\title{
Binarity as a key factor in protoplanetary disk evolution: Spitzer disk census of the $\eta$ Chamaeleontis cluster
}

\author{
J. Bouwman ${ }^{1}$, W. A. Lawson ${ }^{2}$, C. Dominik ${ }^{3}$, E. D. Feigelson ${ }^{5}$, Th. Henning ${ }^{1}$, A. G. G. M. \\ Tielens $^{6}$, L. B. F. M. Waters ${ }^{3,4}$
}

\begin{abstract}
The formation of planets is directly linked to the evolution of the circumstellar (CS) disk from which they are born. The dissipation timescales of CS disks are, therefore, of direct astrophysical importance in evaluating the time available for planet formation. We employ Spitzer Space Telescope spectra to complete the CS disk census for the late-type members of the $\simeq 8$ Myr-old $\eta$ Chamaeleontis star cluster. Of the $15 \mathrm{~K}$ - and M-type members, eight show excess emission. We find that the presence of a CS disk is anti-correlated with binarity, with all but one disk associated with single stars. With nine single stars in total, about $80 \%$ retain a CS disk. Of the six known or suspected close binaries the only CS disk is associated with the primary of RECX 9. No circumbinary disks have been detected. We also find that stars with disks are slow rotators with surface values of specific angular momentum $j=2-15 j_{\odot}$. All high specific angular momentum systems with $j=20-30 j \odot$ are confined to the primary stars of binaries. This provides novel empirical evidence for rotational disk locking and again demonstrates the much shorter disk lifetimes in close binary systems compared to single star systems. We estimate the characteristic mean disk dissipation timescale to be $\sim 5 \mathrm{Myr}$ and $\approx 9$ Myr for the binary and single star systems, respectively.
\end{abstract}

Subject headings: binaries: general - infrared: stars - open clusters and associations: individual ( $\eta$ Cha) - planetary systems: formation - planetary systems: protoplanetary disks stars: pre-main sequence

\footnotetext{
${ }^{1}$ Max Planck Institute for Astronomy, Königstuhl 17, D-69117 Heidelberg, Germany

${ }^{2}$ School of Physical, Environmental and Mathematical Sciences, University of New South Wales, Australian Defence Force Academy, Canberra ACT 2600, Australia

${ }^{3}$ Astronomical Institute "Anton Pannekoek", University of Amsterdam, Kruislaan 403 NL-1098 SJ Amsterdam, Netherlands

${ }^{4}$ Instituut voor Sterrenkunde, KU Leuven, Celestijnenlaan 200B, 3001 Leuven, Belgium

${ }^{5}$ Department of Astronomy \& Astrophysics, Pennsylvania State University, University Park PA 16802

${ }^{6}$ Kapteyn Astronomical Institute, University of Groningen, PO Box 800, 9700 AV Groningen, Netherlands
} 


\section{Introduction}

At birth, protostars are surrounded by circumstellar (CS) gaseous disks that become the natural sites for planet formation. An important constraint for any planet formation scenario is the timescale available during which planet formation can occur, before the CS disks dissipate. Several studies have shown that near and mid-infrared excess flux, indicative of inner CS disks, rapidly declines with $50 \%$ of low-mass stars losing their very inner dust disk within $\sim 3$ Myr (e.g. Haisch et al. 2001; Sicilia-Aguilar et al. 2006). The disappearance of inner disks seems to coincide with the end of accretion, suggesting that both dust and gas disappear on similar timescales (Gulbring et al. 1998; Jayawardhana et al. 2006). The dissipation of the primordial disks in which gas planets could form seems to be completed by $\sim 10$ Myr. However, CS disk evolution is currently poorly-constrained within the $\sim 5-10 \mathrm{Myr}$ age range due to the lack of observationally well-characterized systems.

To study the influence of environment and stellar properties on disk dissipation timescales, nearby young stellar cluster provide ideal targets as many of their stellar properties, their ages and environment can be well constrained. The nearby, coeval $\eta$ Chamaeleontis cluster at a distance of $97 \mathrm{pc}$ and an isochronal age of $\simeq 8 \mathrm{Myr}$ (Mamajek et al. 1999; Lawson et al. 2001; Lyo et al. 2004a; Luhman \& Steeghs 2004) is an excellent laboratory to study disk evolution. The cluster consists of three early-type systems, and 15 low-mass stars with stellar types ranging from K6 to M5 (Lyo et al. 2004a; Luhman \& Steeghs 2004). Lyo et al. (2003) showed that a substantial fraction of the $\eta$ Cha members have an near-IR excess. This was confirmed by Megeath et al. (2005) who observed all late-type members at wavelengths between 3.6-8 $\mu \mathrm{m}$ with the Infrared Array Camera (IRAC) on the Spitzer Space Telescope, detecting disks around $40 \%$ of the stars. Given the age of the $\eta$ Cha cluster, this large disk fraction seems inconsistent with the $\sim 6$ Myr maximum lifetime of inner disks derived by Haisch et al. (2001) from $L$-band observations of younger clusters. This argues against a single dissipation timescale in all environments and/or for all disk radii.

In this paper, we will employ the longer wavelength capabilities of the Infrared Spectrograph (IRS; Houck et al. 2004) onboard Spitzer to firmly establish the census of disks for the 15 low-mass $\mathrm{K}$ - and M-type members of the cluster. We then determine and discuss the influence of binarity on the CS disk dispersal timescales, and on the specific angular momentum distribution of the cluster members. 


\section{Spitzer IRS observations of $\eta$ Cha}

We obtained $7.5-35 \mu \mathrm{m}$ low-resolution $(R=60-120)$ spectra of the $\eta$ Cha cluster members with the IRS spectrograph. Integration times were set such that stellar photospheres could be detected with a signal-to-noise ratio $>5$ across most of the IRS bandpass. Our spectra are based on the droopres products processed through the S13.2.0 version of the Spitzer data pipeline. Partially based on the SMART software package (Higdon et al. 2004), our data was further processed using spectral extraction tools developed for the "Formation and Evolution of Planetary Systems" (FEPS) Spitzer science legacy team. The spectra were extracted using a 6.0 pixel and 5.0 pixel fixed-width aperture in the spatial dimension for the observations with the first order of the short- $(7.5-14 \mu \mathrm{m})$ and the long-wavelength $(14-35$ $\mu \mathrm{m})$ modules, respectively. The background was subtracted using associated pairs of imaged spectra from the two nodded positions along the slit, also eliminating stray light contamination and anomalous dark currents. Pixels flagged by the data pipeline as being "bad" were replaced with a value interpolated from an 8 pixel perimeter surrounding the errant pixel. The low-level fringing at wavelengths $>20 \mu \mathrm{m}$ was removed using the irsfinge package (Lahuis \& Boogert 2003). The spectra are calibrated using a spectral response function derived from IRS spectra and Cohen stellar models for a suite of calibrators provided by the Spitzer Science Centre. To remove any effect of pointing offsets, we matched orders based on the point spread function of the IRS instrument, correcting for possible flux losses. The relative errors between spectral points within one order are dominated by the noise on each individual point and not by the calibration. We estimate a relative flux calibration across an order of $\approx 2 \%$ and an absolute calibration error between orders/modules of $\approx 5 \%$.

Our observations are sensitive to CS disks with substantial inner gaps not radiating at wavelengths $\leq 8 \mu \mathrm{m}$ as covered by IRAC. The maximum wavelength of $35 \mu \mathrm{m}$ of our spectra corresponds to a blackbody temperature of $\sim 90 \mathrm{~K}$. Assuming a $\lambda^{-1}$ dependency for the dust opacity, and a typical stellar temperature and radius of $3500 \mathrm{~K}$ and $1 R_{\odot}$, respectively, dust grains attain a temperature of $90 \mathrm{~K}$ at a radius of about $25 \mathrm{AU}$ from the central star. Therefore our observations should be sensitive to CS disks with a maximum inner gap radius of about this value. Additionally, for circumbinary disks, Artymowicz \& Lubow (1994) argue that the inner edge of circumbinary disks will have a tidal truncation radius of $2.0-2.5 \mathrm{a}$. Given a maximum detection radius of about $25 \mathrm{AU}$, we should be able to detect circumbinary disks in any $\eta$ Cha binary systems with separations $2 a \leq 20$ AU.

In Fig. 1 we show three representative IRS spectra. These examples demonstrate the excellent agreement between the IRS and IRAC data at $8 \mu \mathrm{m}$, both independently calibrated, and between the IRS spectra and the optical photometry-scaled stellar models for diskless systems. From inspection of the spectra, we add RECX 3 and 4 to the disk census, meaning 
that 8 out of 15 (or about $50 \%$ ) of low-mass $\eta$ Cha stars have IRS-detected disks. The newly detected disks lack excesses shortward of $\approx 15 \mu \mathrm{m}$, consistent with the IRAC nondetection and are detected with $>5 \sigma$ certainty at $33 \mu \mathrm{m}$. To quantitatively test for the presence of disks, we calculated the $f(33-13)$ flux ratio derived from the IRS fluxes at 13 $\mu \mathrm{m}$ and $33 \mu \mathrm{m}$, integrating over bandpasses of $1.3 \mu \mathrm{m}$ and $3.3 \mu \mathrm{m}$ centred at each wavelength, respectively. Table 1 lists the $13 \mu \mathrm{m}\left(f_{13}\right)$ and $33 \mu \mathrm{m}\left(f_{33}\right)$ fluxes, and $f(33-13)$ colors for all the late-type $\eta$ Cha stars. Our detection limits at $13 \mu \mathrm{m}$ and $33 \mu \mathrm{m}$ are $\approx 0.15$ and 0.3 mJy, respectively, showing the remarkable sensitivity of the IRS instrument. The six diskless late-type $\eta$ Cha stars with $33 \mu \mathrm{m}$ detections of the stellar photosphere have a $f(33-13)$ color of $0.17 \pm 0.05(1 \sigma)$. In contrast, the weakest disks detected in the transitional disk objects RECX 3 and 4 lack $13 \mu \mathrm{m}$ excesses and have a $f(33-13)$ color of $\simeq 0.6$, while those stars with protoplanetary disks displaying significant disk excesses at both 13 and $33 \mu \mathrm{m}$ have $f(33-13)$ colors exceeding $\simeq 1$.

\section{The influence of binarity on the disk fraction and the angular momentum}

Among the 15 late-type stars, six are binaries with projected separations $\leq 20 \mathrm{AU}$ (column 2, Table 1). Colour-magnitude diagram placement shows nearly half of the late-type stars are elevated by $0.5-0.7$ magnitudes compared to other cluster members of comparable spectral type, indicative of binary systems with near-equal luminosity components (Lyo et al. 2004b). Of these stars, RECX 1 and 9 are resolved with a projected spatial separation of $\simeq 20 \mathrm{AU}$, following speckle and AO imaging surveys for close companions (Köhler \& PetrGotzens 2002; Brandeker et al. 2006). RECX 12 is an unresolved binary with an inferred separation of $\approx 4 \mathrm{AU}$ (Brandeker et al. 2006), and has a dual-periodicity (1.3 d and $8.5 \mathrm{~d}$ ) light curve measured during a photometric survey for starspot-modulated variability (Lawson et al. 2001). From $v \sin i$ measurements of RECX 12 (Covino et al. 1997; Jayawardhana et al. 2006), we assume the 1.3-d mode to be associated with the primary. RECX 7 is a 2.6- $\mathrm{d}$ period, dual-lined spectroscopic binary of mass ratio 2.3:1 and separation $\approx 0.1 \mathrm{AU}$ (Lyo et al. 2003). Given the spatial resolution of the Brandeker et al. (2006) study, the projected binary separations for the ECHA J0836.2-7908 and J0838.9-7916 systems are <4 AU. The cluster has a deficit of wide binaries at projected separations $>20-50$ AU (e.g. Brandeker et al. 2006).

The disk frequency is summarized in Fig. 2. Our spectra reveal a remarkable divergence in disk presence as a function of binarity in the cluster's late-type population. Of the eight detected disks, all but one are associated with single stars. With nine single stars in total, about $80 \%$ have a CS disk. Of the six known or suspected close binaries, only RECX 9AB 
has a CS disk associated with it. The presence of this disk, likely a circumprimary disk, was already inferred from ground-based $L$-band photometry (Lyo et al. 2003), $\mathrm{H} \alpha$ spectroscopic measurements of disk accretion (Lawson et al. 2004), and IRAC photometry (Megeath et al. 2005). No circumbinary disks are detected. A non-parametric Mann-Whitney two-sample $U$-test indicates that the probability that the singles and binaries are drawn from the same parent sample is $P=0.03$, i.e. CS disks are under-represented in $\eta$ Cha binaries with $>2 \sigma$ significance. We surmise that the last "remaining" disk in a binary exists principally because the physical separation of RECX $9 \mathrm{AB}$ greatly exceeds the projected $\simeq 20 \mathrm{AU}$ separation, given that no significant change in spatial separation or position angle was detected between the surveys of Köhler \& Petr-Gotzens (2002) and Brandeker et al. (2006). The evolution of the disk around RECX 9A, therefore, effectively follows that of a disk in a single star system. The $f(33-13)$ color of the RECX 9 disk suggests that the outer disk extends to at least 20 AU. The presence of an extended disk in combination with a circumprimary tidal truncation radius of $\approx 0.4 a$ (Artymowicz \& Lubow 1994) implies a physical binary separation $2 a \geq 50$ AU. If the evolution of RECX 9 is treated as a single star then a $U$-test gives $P=0.003$, indicating that the evolution of single stars or binaries with separations equal or larger than that of RECX 9 is significantly different from the evolution of close binary systems.

To estimate a typical disk disappearance timescale, we assume a rectangular probability distribution for the disk to dissipate around a mean timescale $t_{d}$ and width $2 \sigma$. As constraints we assume that at $t=0 \mathrm{Myr}$ all systems have disks, and that by $t=12 \mathrm{Myr}$ (the age of the $\beta$ Pic moving group) all disks have dissipated. We find for the binary and single star members, respectively, $t_{d}=5 \pm 5 \mathrm{Myr}$ and $t_{d}=9 \pm 3 \mathrm{Myr}$. If for our widest binary system, RECX 9AB, single star evolution is more applicable, the derived $t_{d}$ for disks in close binary systems could be even shorter.

Herbst \& Mundt (2005) analysed the rotational evolution of samples of solar-like PMS stars. Their key conclusion was that around half of PMS stars lose significant surface angular momentum in the first 5-6 Myr owing to rotational coupling between star and disk. During this phase, spin-up of the star is prevented as it evolves towards lower luminosity, because angular momentum is transferred to the CS disk. The other half of PMS stars evolve at almost constant surface angular momentum, having lost their disks within the first few million years. These two groups of PMS stars are believed to evolve to form the slowly- and rapidly-rotating groups of young main sequence stars, respectively.

We calculated the magnitude of the specific surface angular momentum $j$ (surface angular momentum per unit mass) for the 12 late-type cluster members with rotation periods and photometry from Lawson et al. $(2001,2002)$ and precise spectral types from Lyo et al. (2004a). We adopt dwarf temperature and bolometric correction scales to calculate stel- 
lar luminosity and radii, with compensation applied for companion stars within the binary systems based upon observed light and radial velocity ratios. For binaries, we assume the observed rotation period is associated with the primary star. In Table 1 we express the derived $j$ values in solar units, where $j_{\odot}=9.5 \times 10^{15} \mathrm{~cm}^{2} \mathrm{~s}^{-1}$. In Fig. 3 we plot the cluster's $j$ distribution for various groupings of cluster objects. We see that all the high- $j$ systems are the primaries of $\eta$ Cha binary systems. With a mean $j=22$ for binary primaries, this group differs significantly from the single stars with a mean $j=6$. A $U$-test gives a probability that the single stars and primaries are drawn from the same parent sample of only $P=0.02$. The comparison would have been even more extreme if we had not compensated for the presence of secondary stars. For uncorrected binaries having a mean $j=37$, the $U$-test gives a probability of $P=0.004$.

We thus provide novel empirical evidence for rotational disk locking, supporting models of star-disk magnetic coupling (e.g. Bouvier et al. 1997; Küker et al. 2003). We concur with Herbst \& Mundt (2005) that high- $j$ stars are freed from any form of locking mechanism. In the $\eta$ Cha cluster, none of the high- $j$ stars have CS disks. We additionally add the result that the high- $j$ tail in $\eta$ Cha consists entirely of the primaries of binary stars. This again argues that disk lifetimes in binary systems are shorter than those in single star systems. The $j$ samples of Herbst \& Mundt (2005) are not corrected for binarity. From our results for the $\eta$ Cha cluster, we surmise that the high- $j$ tail of their distributions are populated with, and possibly dominated by, diskless binary systems.

\section{Conclusions}

The link between disk presence, angular momentum and binarity may have profound astrophysical importance in evaluating the time available for planet formation, as this is determined by the dissipation timescale of protoplanetary disks. The high angular momentum and paucity of disks in $\eta$ Cha binaries indicates a characteristic disk lifetime considerably less than the cluster's age of $\simeq 8 \mathrm{Myr}$. This is in contrast to the $\eta$ Cha singles, where the high disk fraction implies that their disk evolutionary timescale is comparable to, or slightly greater than, the age of the cluster. Using a simple statistical approach, we estimate a mean disk dispersion timescale of $\sim 5 \mathrm{Myr}$ and $\approx 9$ Myr for close binary and single star systems, respectively. Our results suggest that the correct evaluation of the disk fraction in PMS groups, and consequently the characteristic timescale for disk longevity during the PMS phase, critically depends on knowledge of binarity within a given PMS population.

We cannot rule out that disk dissipation timescales could also depend on stellar mass, as our sample only consists of late K- and M-type stars. Also, the derived timescales depend 
upon the detection wavelength, reflecting that disk dissipation can have a radial dependence. However, even if we limit our detections to those stars with IRAC excesses, dissipation timescale for single stars only shortens by $\sim 10 \%$ to $8 \pm 4 \mathrm{Myr}$. In any case, our results imply that the assumption of a single timescale for disk dissipation is not correct. The rapid decline of the disk fraction in the first few Myr as inferred by several near- and mid-IR studies, could be dominated by the dissipation of disks in close binary systems. The slower dissipation of disks around single stars could be the explanation for the long-lived disks seen in older PMS clusters such as $\eta$ Cha.

The strong tidal torques exerted on disks in close binary systems will have a negative impact on the efficiency of planet formation (e.g. Kley 2001; Mayer et al. 2005). Indeed, while $\sim 25 \%$ of exo-planets are detected in binaries, no planets have been found in binaries with projected separations $<20$ AU (Raghavan et al. 2006), though at this point observational selection effects can not be ruled out. Our results imply that in close binary systems the time available for planet formation is considerably shorter than in single star systems, which could severely inhibit the formation of planets. The apparent lack of planets in close binaries, therefore, might reflect the shorter disk dissipation timescale in binary systems.

EDF and AGGMT acknowledge support from Spitzer GO grant No. 3508 (PI WAL). WAL acknowledges support from UNSW@ADFA Faculty Research Grant Programs 2005 and 2006. JB and ThH acknowledge support from the EU HPR network contract No. HPRN-CT-2002000308. We thank Leen Decin, University of Leuven, for the stellar models.

\section{REFERENCES}

Artymowicz, P., \& Lubow, S. H. 1994, ApJ, 421, 651

Bouvier, J., Forestini, M., \& Allain, S. 1997, A\&A, 326, 1023

Brandeker, A., Jayawardhana, R., Khavari, P., Haisch, K. E., Jr, \& Mardones, D. 2006, astro-ph/0608352

Covino, E., Alcalá, J. M., Allain, S., Bouvier, J., Terranegra, L., \& Krautter, J. 1997, A\&A, 328,187

Gullbring, E., Hartmann, L., Briceno, C., \& Calvet, N. 1998, ApJ, 492, 323

Haisch, K. E., Jr, Lada, E. A., \& Lada, C. J. 2001, ApJ, 553, L153

Higdon, S. J. U. et al. 2004, PASP, 116, 975 
Herbst, W., \& Mundt, R. 2005, ApJ, 633, 967

Houck, J. R., Roellig, T. L., Van Cleve, J., Forrest, W. J., Herter, T. L., Lawrence, C. R., Matthews, K., Reitsma, H. J., et al. 2004, ApJS, 154, 18

Jayawardhana, R., Coffey, J., Scholz, A., Brandeker, A., \& van Kerkwijk, M. H. 2006, astro$\mathrm{ph} / 0605601$

Kley, W. 2001, IAU Symposium, 200, 511

Köhler, R., \& Petr-Gotzens, M. G. 2002, AJ, 124, 2899

Küker, M., Henning, Th., \& Rüdiger, G. 2003, ApJ, 589, 397

Lahuis, F., \& Boogert, A. 2003 , in SFChem 2002: Chemistry as a Diagnostic of Star Formation, Edited by Charles L. Curry \& Michel Fich, NRC Press, Ottawa, Canada, 335 .

Lawson, W. A., Crause, L. A., Mamajek, E. E., \& Feigelson, E. D. 2001, MNRAS, 321, 57

Lawson, W. A., Crause, L. A., Mamajek, E. E., \& Feigelson, E. D. 2001, MNRAS, 322, L29

Lawson, W. A., Lyo A.-R., \& Muzerolle J. 2004, MNRAS, 351, L39

Luhman, K. L., \& Steeghs, D. 2004, ApJ, 609, 917

Lyo A.-R., Lawson, W. A., Mamajek, E. E., Feigelson, E. D., Sung, E.-C., \& Crause, L. A. 2003, MNRAS, 338, 616

Lyo A.-R., Lawson, W. A., \& Bessell M. S. 2004, MNRAS, 355, 363

Lyo A.-R., Lawson, W. A., Feigelson, E. D., \& Crause, L. A. 2004, MNRAS, 347, 246

Lyo A.-R., Song, I., Lawson, W. A., Bessell M. S., \& Zuckerman, B. 2006, MNRAS, 368, 1451

Mamajek, E. E., Lawson, W.A., \& Feigelson, E. D. 1999, ApJ, 516, L77

Megeath, S. T., Hartmann, L., Luhman, K. L., \& Fazio, G. G. 2005, ApJ, 634, 113

Mayer, L., Wadsley, J., Quinn, T., \& Stadel, J. 2005, MNRAS, 363, 641

Raghavan, D., Henry, T. J., Mason, B. D., Subasavage, J. P., Jao, W.-C., Beaulieau, T. D., \& Hambley, N. C. 2006, ApJ, 646, 523 
Sicilia-Aguilar, A. et al. 2006, ApJ, 638, 897

This preprint was prepared with the AAS LATEX macros v5.2. 
Table 1. Summary of binary, disk and rotational properties for late-type $\eta$ Cha stars

\begin{tabular}{|c|c|c|c|c|c|c|}
\hline $\begin{array}{l}\text { Star } \\
\text { (1) }\end{array}$ & $\begin{array}{c}\text { Binary } \\
\text { (2) }\end{array}$ & $\begin{array}{l}\text { Disk } \\
\text { (3) }\end{array}$ & $\begin{array}{c}f_{13} \\
(\mathrm{mJy}) \\
(4)\end{array}$ & $\begin{array}{c}f_{33} \\
(\mathrm{mJy}) \\
(5)\end{array}$ & $\begin{array}{c}f(33-13) \\
(6)\end{array}$ & $\begin{array}{c}j \\
\left(j_{\odot}\right) \\
(7)\end{array}$ \\
\hline RECX 1 & $0.2^{\prime \prime}$ & $\mathrm{n}$ & 39.9 & 7.0 & 0.18 & 28.5 \\
\hline RECX 3 & & $\mathrm{y}$ & 6.1 & 3.5 & 0.57 & 5.0 \\
\hline RECX 4 & & $\mathrm{y}$ & 11.5 & 7.6 & 0.66 & 5.2 \\
\hline RECX 5 & & $\mathrm{y}$ & 21.0 & 68.0 & 3.24 & 4.2 \\
\hline RECX 6 & & $\mathrm{n}$ & 6.6 & 0.9 & 0.14 & 13.1 \\
\hline RECX 7 & $0.001^{\prime \prime}$ & $\mathrm{n}$ & 27.2 & 6.9 & 0.25 & 21.0 \\
\hline RECX 9 & $0.2^{\prime \prime}$ & $\mathrm{y}$ & 42.5 & 49.1 & 1.16 & 11.1 \\
\hline RECX 10 & & $\mathrm{n}$ & 10.4 & 1.1 & 0.11 & 1.9 \\
\hline RECX 11 & & $\mathrm{y}$ & 228.7 & 222.4 & 0.97 & 14.5 \\
\hline RECX 12 & $0.04^{\prime \prime}$ & $\mathrm{n}$ & 16.0 & 2.0 & 0.13 & 25.2 \\
\hline ECHA J0836.2-7908 & $<0.04^{\prime \prime}$ & $\mathrm{n}$ & 1.7 & 0.0 & 0.00 & $\cdots$ \\
\hline ECHA J0838.9-7916 & $<0.04^{\prime \prime}$ & $\mathrm{n}$ & 3.2 & 0.5 & 0.17 & $\cdots$ \\
\hline ECHA J0841.5-7853 & & $\mathrm{y}$ & 9.0 & 11.3 & 1.25 & 2.7 \\
\hline ECHA J0843.3-7905 & & $\mathrm{y}$ & 155.8 & 267.8 & 1.72 & 2.5 \\
\hline ECHA J0844.2-7833 & & $\mathrm{y}$ & 7.2 & 16.6 & 2.30 & $\cdots$ \\
\hline
\end{tabular}

Note. - Table columns: Col 1. Common star name. Col 2. Projected or inferred separations for the binaries (see $\S 3.1$ for details). Col 3. Presence of disk. Col 4. Spitzer IRS $13 \mu \mathrm{m}$ flux $f_{13}$. Col 5. Spitzer IRS $33 \mu \mathrm{m}$ flux $f_{33}$. Col 6. $f(33-13)$ color ratio $=f_{33} / f_{13}$. Col 7. Surface specific angular momentum $j$ in solar units, where $j_{\odot}=9.5 \times 10^{15} \mathrm{~cm}^{2} \mathrm{~s}^{-1}$. For RECX 1, 7, 9 and 12 , we list the $j$ value for the primary. 


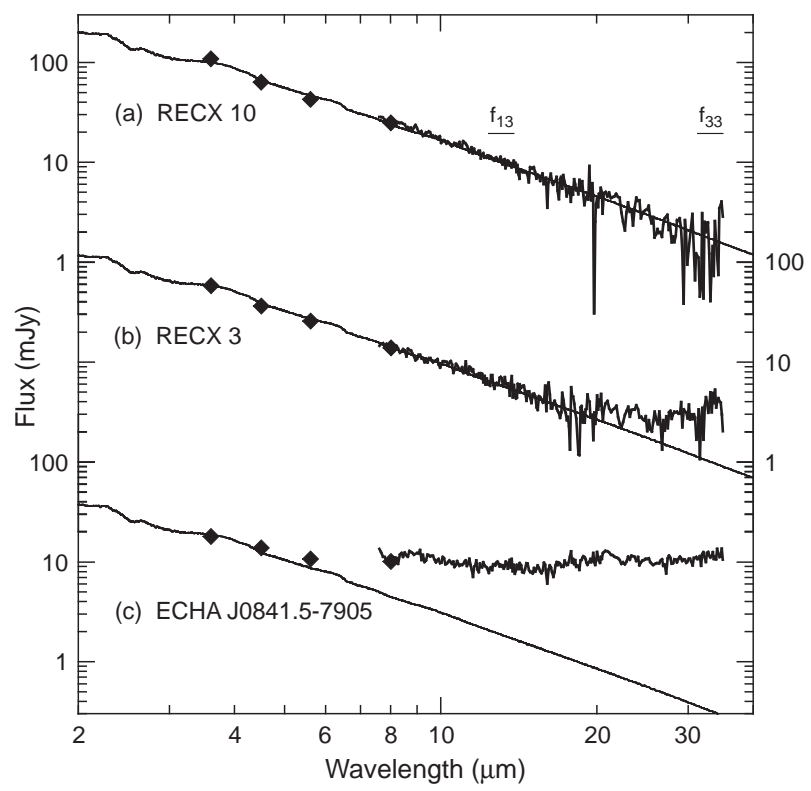

Fig. 1.- Representative Spitzer IRS spectra of $\eta$ Cha cluster stars. Bold lines are the IRS spectra, diamonds are IRAC photometry, while the thin lines are MARCS stellar models generated using precise spectral types for the stars then scaled to optical photometry. Panel (a) shows the diskless spectrum of RECX 10. Panel (b) shows the spectrum of RECX 3, a transitional disk object with the weakest detected IRS disk with $f_{33}=3.5 \mathrm{mJy}$. Panel (c) shows ECHA J0841.5-7853 with a 10 mJy disk displaying the typical spectral properties of a protoplanetary disk. In panel (a) we indicate the $13 \mu \mathrm{m}$ and $33 \mu \mathrm{m}$ filter bands used to define the $f(33-13)$ colors in $\S 2$ and Table 1. 


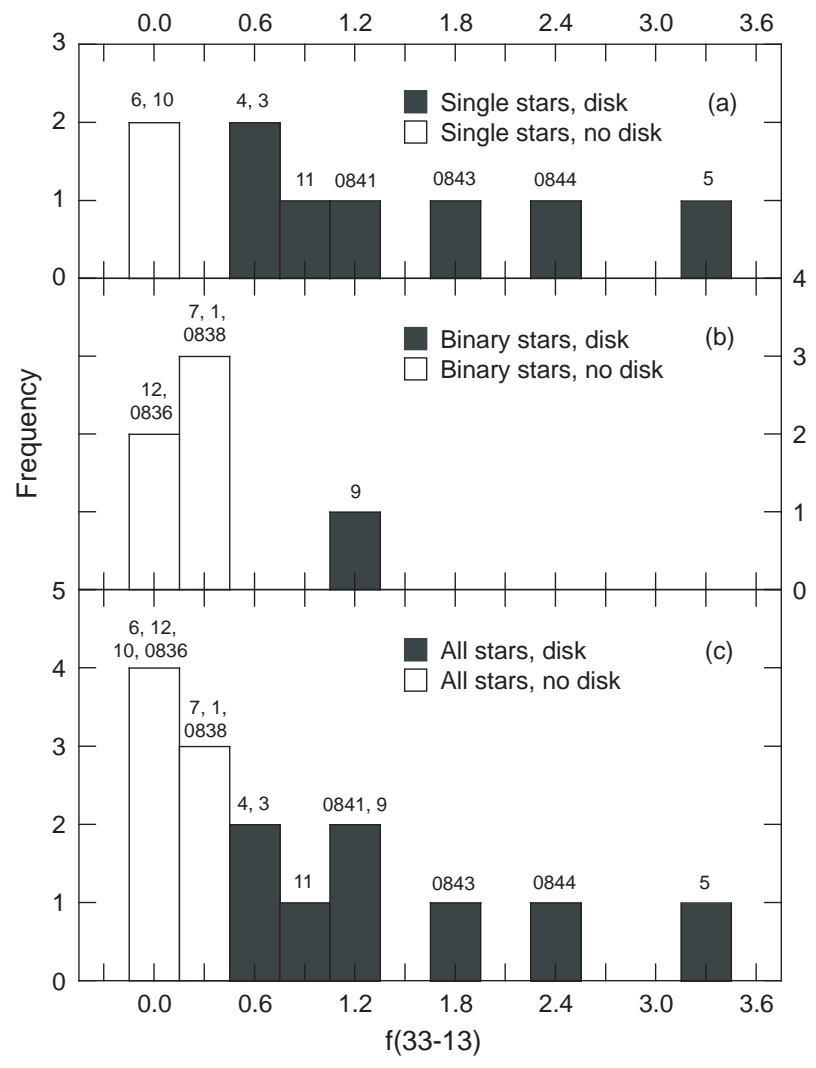

Fig. 2.- Distributions of the $f(33-13)$ color for the late-type $\eta$ Cha stars. In this figure, individual stars identified by abbreviated labels, e.g. RECX 11 is labeled '11' and ECHA J0843.3-7905 is labeled '0843'. Panel (a) and (b) plot the colors of single stars and binary stars with (without) disks, respectively, while in panel (c) we merge the $f(33-13)$ colors for the entire late-type population. 


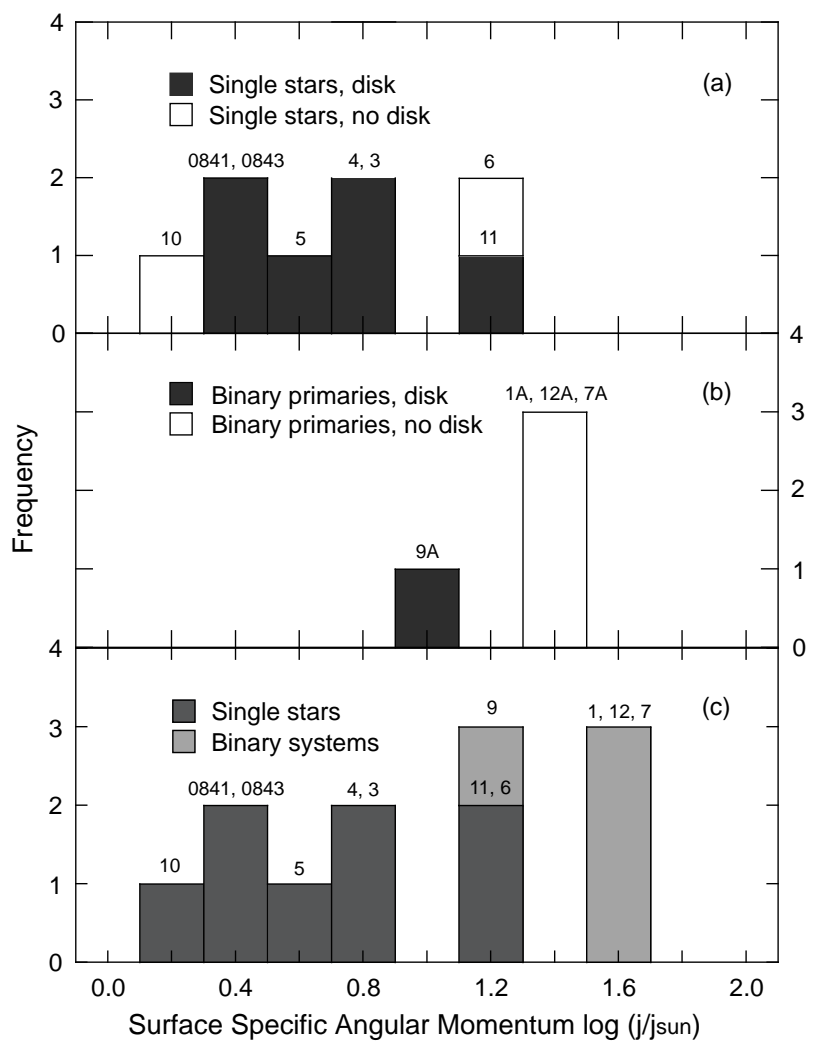

Fig. 3.- Distributions of surface specific angular momentum $j$ in solar units. As in Fig. 2, individual stars are identified by abbreviated labels, with the addition that binary primaries are further denoted, e.g. RECX $12 \mathrm{~A}$ is labeled ' $12 \mathrm{~A}$ '. Panel (a) plots $j$ values for single stars with (without) disks, while panel (b) is a similar distribution for binary primaries. In panel (c) we assemble the $j$ values for the cluster without compensation for binary companions. Panel (c) is the standard representation for the $j$ distribution where no knowledge is assumed about binary properties. 